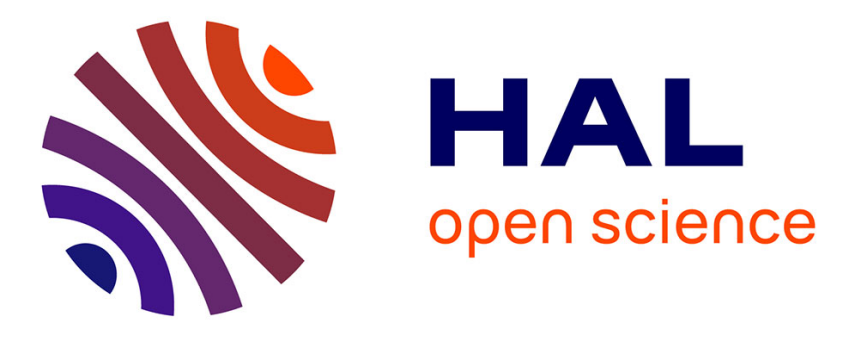

\title{
A Novel Task for Studying Memory of Occasional Events in Rats
}

Marina Allerborn, Alexandra Gros, Belkacem Messaoudi, Damien Gervasoni, Samuel Garcia, Marc Thevenet, Serge Laroche, Alexandra Veyrac, Nadine Ravel

\section{To cite this version:}

Marina Allerborn, Alexandra Gros, Belkacem Messaoudi, Damien Gervasoni, Samuel Garcia, et al.. A Novel Task for Studying Memory of Occasional Events in Rats. Bio-protocol , 2016, 6 (5), 10.21769/BioProtoc.1740 . hal-01896058

\section{HAL Id: hal-01896058 https://hal.science/hal-01896058}

Submitted on 15 Oct 2018

HAL is a multi-disciplinary open access archive for the deposit and dissemination of scientific research documents, whether they are published or not. The documents may come from teaching and research institutions in France or abroad, or from public or private research centers.
L'archive ouverte pluridisciplinaire HAL, est destinée au dépôt et à la diffusion de documents scientifiques de niveau recherche, publiés ou non, émanant des établissements d'enseignement et de recherche français ou étrangers, des laboratoires publics ou privés. 


\title{
A Novel Task for Studying Memory of Occasional Events in Rats \\ Marina Allerborn ${ }^{1 \#}$, Alexandra Gros ${ }^{2 \#}$, Belkacem Messaoudi ${ }^{1}$, Damien Gervasoni ${ }^{1}$, Samuel Garcia $^{1}$, Marc Thevenet ${ }^{1}$, Serge Laroche ${ }^{2}$, Alexandra Veyrac ${ }^{1}$ and Nadine Ravel ${ }^{1 *}$
}

\author{
${ }^{1}$ Centre de Recherche en Neurosciences de Lyon, Université de Lyon, Lyon, France; \\ ${ }^{2}$ Paris-Saclay Neuroscience Institute, Université Paris-Saclay, Université Paris-Sud, CNRS, \\ Orsay, France \\ \#Contributed equally to this work \\ *For correspondence: nadine.ravel@cnrs.fr
}

[Abstract] Episodic memory has been defined in humans as the conscious recollection of unique personal past experiences often occurring singly during daily life, including remembrance of what happened, where and when it happened (Tulving, 1972). Here, we propose and describe in details a novel protocol we recently used to test the ability of rats to form and recollect episodic-like memory of previously encountered occasional episodes (Veyrac et al., 2015). During these episodes, the animals are briefly exposed to sets of specific odor-drink associations (what happened) encountered in specific locations (where it happened) within different multisensory enriched environments (in which context/occasion it happened). Memory of the episodes can be tested at relatively short (24 h) or much longer (24 d) delays in either a low or high interfering retrieval situation. This novel paradigm brought evidence for individual memory profiles of recall performance that might be correlated to different aspects of brain functional networks. More generally, it offers novel possibilities to explore cellular and network mechanisms that underlie memory of past events and memory dysfunction in brain pathologies.

\section{Materials and Reagents}

1. Subjects

All experiments were conducted in accordance with European guidelines for care of laboratory animals (2010/63/EU) and received approval from the Lyon 1 University Ethics Committee (permission DR2015-46). Adult male Long-Evans rats (Charles River Laboratories) aged 7-8 weeks ( $300-350 \mathrm{~g})$ at the start of the water deprivation protocol, are housed in groups of 2-4 per cage and kept in an environment with controlled temperature and humidity under a 12/12 h light/dark cycle with food ad libitum. Experiments are conducted during the light period.

Note: So far these experiments have not been conducted with other strains of rats or mice, but we assume that usage of other strains shouldn't introduce major difficulties to carry out the experiments. Nevertheless, according to the fact that contexts are characterized by visual stimulation, working with albinos rats might be more 
challenging. Regarding mice, some adjustments to the experimental device would be required.

2. Odorants

In order to be delivered in a fully controlled manner through a new generation of olfactometer (Sezille et al., 2013), each odorant is introduced in a U-shaped Pyrex ${ }^{\circledR}$ tube (volume: $10 \mathrm{ml}$; length: $50 \mathrm{~mm}$; external diameter: $14 \mathrm{~mm}$ ) (VS technologies) filled with microporous granules. All odors used in these experiments were obtained from Sigma-Aldrich, France (see a-f below).

a. Geraniol (Sigma-Aldrich, catalog number: 163333) 20\% of saturated vapor pressure

b. Eugenol (Sigma-Aldrich, catalog number: E51791) 18\% of saturated vapor pressure

c. (S)-(+)-Carvon (Sigma-Aldrich, catalog number: 435759) 35\% of saturated vapor pressure $=$ Odor $\mathrm{A}$

d. Isoamylacetate (Sigma-Aldrich, catalog number: W205508) $15 \%$ of saturated vapor pressure $=$ Odor $B$

e. Trans-Anethole (Sigma-Aldrich, catalog number: 117870) 30\% of saturated vapor pressure $=$ Odor $\mathrm{C}$

f. Citral (cis+trans) (Sigma-Aldrich, catalog number: W230308) 20\% of saturated vapor pressure $=$ Odor $D$

Note: Before the start of experiments, between 2 and $4 \mathrm{ml}$ of pure odor solution are introduced gradually on several consecutive days into the $U$ Shaped Glass Tubes until the microporous granules appear saturated (Figure 1) (for the experiments, the odors should be at saturated vapor pressure in the tube, at the same time in order to prevent olfactometer pollution it is important to avoid the accumulation of liquid at the bottom of the tube). The percentage of saturated vapor pressure introduced into the airflow is adjusted for each odor individually by the experimenter. The objective is to obtain an intensity that is perceived by the animal but moderate enough to prevent any avoidance behavior. For the different odors used in pairs, we also try to equalize their perceived intensity. The choice of these particular odors and concentrations was mainly determined by previous experiments in the lab showing their easy discriminability and the absence of their natural attractiveness or repulsiveness to the rats at the concentrations used (Martin et al., 2004; Courtiol et al., 2014; Torquet et al., 2014). 


\section{bio-protocol}

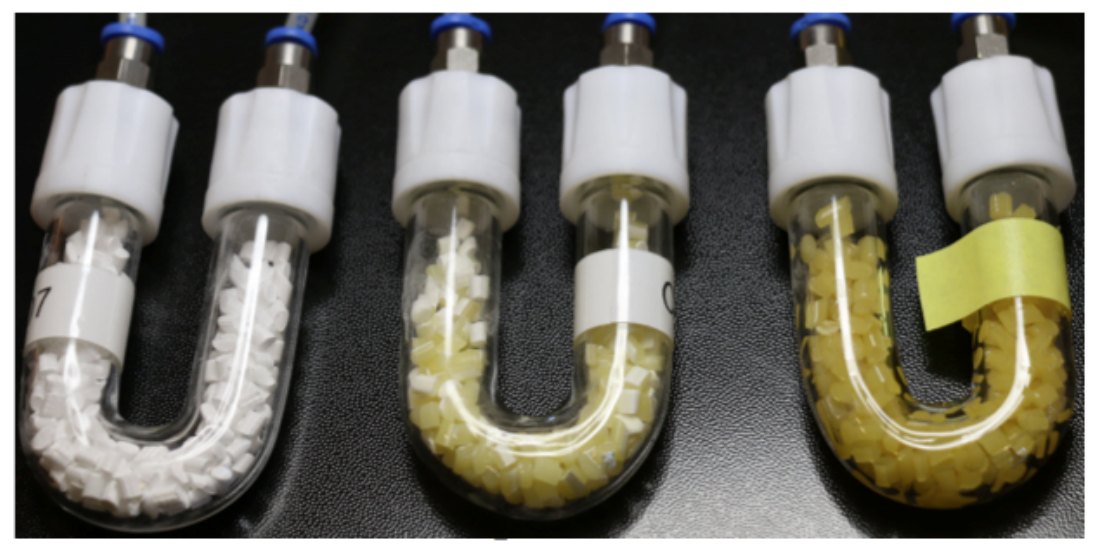

Figure 1. A photo of the U-shaped Pyrex tube filled with microporous granules showing different levels of saturation with Carvon odor solution. From left to right: pure granules without any added odor solution; partly saturated granules; fully saturated granules as used for the experiments.

3. Drinking solutions

a. $6 \%$ Sucrose in solution is used as positive reinforcement (Sigma-Aldrich, catalog number: number: 84097)

b. $0.06 \%$ Quinine hydrochloride dehydrate in solution is used as negative reinforcement (Sigma-Aldrich, catalog number: Q1125)

\section{Equipment}

1. Apparatus (EPISODICAGE)

The experimental cage is a PVC rectangular box $(60 \times 35 \times 40 \mathrm{~cm})$ equipped with 4 devices for delivery of different odor and drinking solutions (Figure 2A) (Belkacem Messaoudi). On the two opposing walls, at $5 \mathrm{~cm}$ distance from each corner of the box, the cage contains an arrangement of odor and drinking ports. The odor port is a round indentation into the wall ( $5 \times 5 \mathrm{~cm}$ in size), with a drinking port (small hole, $1 \times 1 \mathrm{~cm}$ in size) placed $1 \mathrm{~cm}$ below the odor port through which a drinking pipette can be inserted into the cage (Figure 1B). 


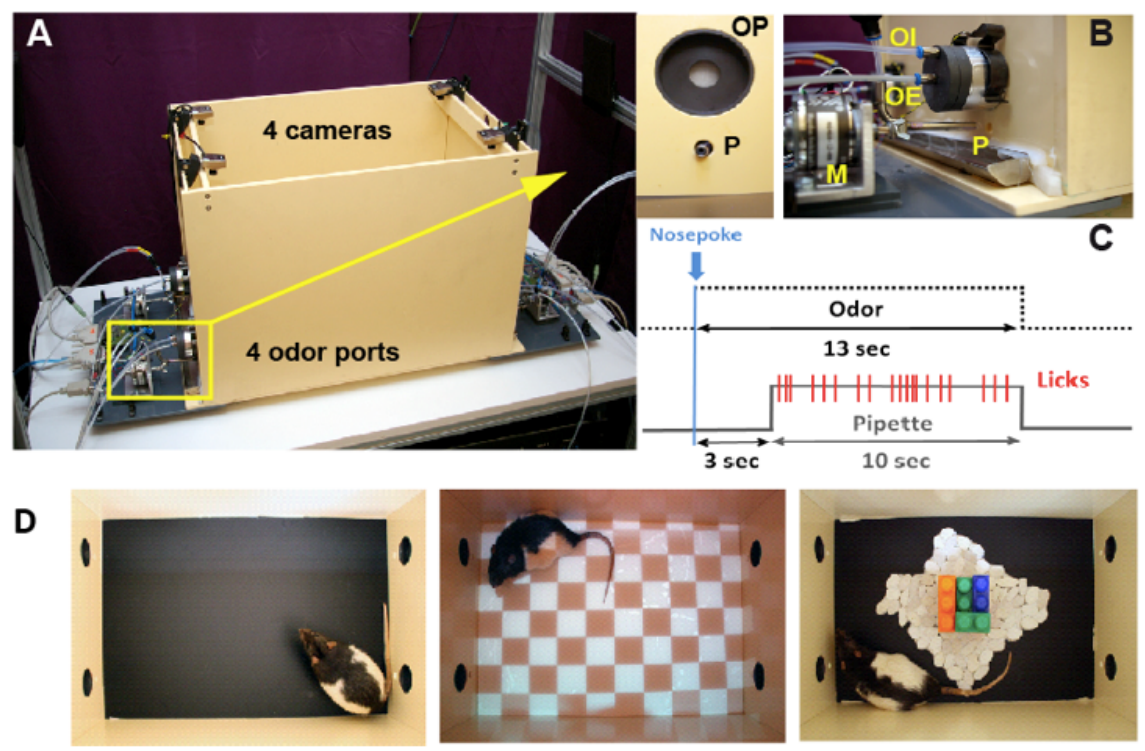

Figure 2. The EPISODICAGE. A. General view of the experimental cage showing the four cameras installed above each of the four odor ports. B. Details of an odor port $(\mathrm{OP})$ with associated drinking pipette $(\mathrm{P})$, view from the inside (left figure) or the outside (right figure) of the cage: odor injection (OI) and odor extraction (OE), motor (M), which allows introducing and withdrawing the pipette from the cage. C. Each nose poke first triggers an odor delivery for $13 \mathrm{sec}$. Three seconds after odor initiation, a pipette containing various drinking solutions (water, sugar or quinine) is introduced into the cage for $10 \mathrm{sec}$ until the odor stimulation switches off. Each lick on the pipette made by the rat is detected and recorded by the computer devoted to the control of the experimental cage. Each trial starts with the nose poke and ends with withdrawal of the pipette and odor switch off. D. On the left, appearance of the cage in the version used in routine sessions. The two other pictures illustrate how the appearance of the cage can be modified by projecting visual patterns on the ground (center picture) or introducing objects (right picture). Figure adapted from Veyrac et al. (2015).

a. Odor port

When a rat makes a nose poke into the port, a capacitance change is detected by the system that immediately triggers for $13 \mathrm{sec}$ the introduction of odor-saturated vapor into a constant airflow (air flow overall: 1 liter/min). The proportion of odorized air and the quality of the odorant are controlled through a custom made olfactometer connected to the odor port (OI) (Figure 1B). In a given experimental session, five different odors can be delivered at each of the four odor ports. A venturi-based system of aspiration allows extraction of the odorized air through a separate channel, so that the odor remains limited to the port (OE) (Figure 1B). A permanent compensation of $\mathrm{Ol}$ and $\mathrm{OE}$ prevents any change in overall air pressure due to odor switch. 
Note: Rats are highly sensitive to changes in air pressure. To prevent any behavioral response to mechanical stimulation, air pressure must be kept at the same level during the presence and absence of odors.

b. Drinking port

Each movable drinking pipette is connected to a pump. The pipette is introduced into the cage $3 \mathrm{sec}$ after the first nose poke of the animal and is withdrawn from the cage $10 \mathrm{sec}$ later simultaneously with termination of odor release (Figure 1C). This ensures that the odor stimulation is delivered during the entire liquid consumption. A second capacitance change sensor integrated into the drinking pipette detects every single lick of the animal and triggers the pump which in turn delivers a calibrated amount of 8-9 $\mu \mathrm{l}$ of drinking solution. The licks made by the rats are recorded as text files for later analysis of the results.

Note: The amount of liquid delivered upon each lick from the drinking ports must be equal between the ports (the variation should be within $8 \pm 1 \mu l$ ), in order to avoid any preference of the animals for a particular port.

Different drinking solutions can be delivered by the system, water, sucrose or quinine solutions according to the different phases of the protocol and the odor-port configurations. After each trial and retraction of the pipette, the drinking solution device is purged with the solution of the following trial.

Note: Purge duration is set in a way that allows the complete wash out of the drinking solution of the last trial. This was verified through addition of ink into the water before the start of experiments.

2. Items for contextual enrichment during episodes

Acoustic context

Different types of sounds can be played through two loudspeakers symmetrically placed above the experimental arena to enhance the discriminability of the episodes: nature sounds, bird songs, piano music ...etc. The general guideline for choosing these sounds was their discriminability as validated in spectrograms and the animals/experimenter's well-being.

Tactile and visual context

Different types of floors can be used with distinct tactile and visual characteristics (Figure 1D). As color vision in rats is poor, combinations of high-contrast black and white coloring of materials are preferred. Additionally, visual patterns can also be displayed on the floor by a video projector and various objects can be placed into the box.

3. Video recording

Five cameras are used to monitor precisely the behavior of the rats, one placed centrally above the experimental box and four others each placed above the four odor ports. The position of the rat is detected online via a video tracking software from the signal recorded from the central camera. When the animal approaches a port, the 


\section{biö-protocol}

corresponding camera is selected for signal acquisition. This allows us to save space occupied by the video files.

\section{Software}

1. VOLCAN (Marc Thevenet)

\section{Procedure}

All the essential steps of the protocol are summarized in Figure 3. Prior to the start of the shaping procedures, rats are habituated to the experimenter and water deprivation is established.

A. Water deprivation protocol

During the experiments, and most critically during the shaping period, the animals have to be motivated enough to activate the drinking device. This requires a controlled reduction of their access to water in the animal housing facility. It must not be too severe to preserve the animal in a good physiological state, but sufficient enough to keep them motivated in a controlled and stable way. One week after their arrival in the animal facility, when the animals are habituated to their new housing environment, we quantify daily ad lib water consumption for each individual cage by measuring the bottle weight before and after drinking. Water restriction is introduced progressively one week later: during the first $2 \mathrm{~d}$ of deprivation, a drinking pipette is accessible in their home cage for $2 \mathrm{~h}$ in the morning (between 9 and $11 \mathrm{am}$ ) and in the evening (4 to $6 \mathrm{pm}$ ) respectively, then the access is reduced to $1 \mathrm{~h}$ during the next $2 \mathrm{~d}$ and to 40 min during the following $7 \mathrm{~d}$. All along this period, we control that the total amount of water consumed is close to the previous consumption during the ad lib period and consequently adjust the duration of the evening access. Additionally, the weight of the animals is periodically checked to ensure that it remains steady $( \pm 5 \%)$ across the entire protocol. During behavioral experiments, if rats are tested in the morning, they receive water for 20-40 min only in the evening, but if they are tested in the afternoon, they receive water during $\sim 5 \mathrm{~min}$ in the morning and for 20-40 min in the evening. Moreover, evening access to water may be adjusted all along the experiments depending on the amount of water consumed by the animals in the experimental arena.

Note: Within a $20 \mathrm{~min}$ period, rats are expected to perform at least 10 trials. For animals that do not perform the expected number of trials, and do not exhibit other signs than a lack of motivation, the access time to drinking water is reduced (e.g., from 40 to $20 \mathrm{~min}$ ). Additionally, it is helpful to decrease the amount of drinking time on the day which precedes the first episode to increase the motivation of the rats and overcome stress which arises through exposure to a novel situation.

Water consumption of the animals when water restriction has been established is 
estimated to around $20 \mathrm{ml} / \mathrm{d}$. Upon water or sugar delivery, the rats make approximately 60 licks per trial, drinking $8 \mu \mathrm{l}$ solution per lick, thus consuming around $10 \mathrm{ml}$ in 20 trials. The time of free water access in the evening during the experimental period can therefore be adjusted in such a way that rats can consume at least $10-15 \mathrm{ml}$ when the bottle is accessible for drinking.

For the long-term test performed 24 days after the last episode presentation, water deprivation is interrupted at the end of the episode presentations and reinstalled one week prior to the test. It should also be slightly stricter than during exposure to the episodes, because by that time the rats spontaneously decrease their water consumption.

B. Shaping

Note: Because the time during which rats are exposed to the episodes is relatively limited, it is essential to minimize stress as much as possible in order to maximize behavioral exploration during episodes. Additionally, during shaping the protocol is adapted to each individual rat in order to start the episodic phase with a relatively homogenous group of rats in terms of their behavioral exploration.

Habituation to the experimenter

One week after arrival of the rats the experimenter starts handling and playing with the rats for 5 min twice a day (once in the morning and once in the afternoon), first in the animal facility and later in the experimental room, which continues for 2-3 weeks until the start of the experiment. Handling should also be done for one week before the long-term test to ensure that the rats get used again to the experimenter (this time only in the animal facility).

Note: Preferably the same person should carry out the experiments, since it is an episodic memory task and the experimenters might be associated to the context of the episode by the animals. If several experimenters are doing the experiments, one should then pay attention that the same experimenter is performing the episode session and corresponding test (e.g., Episode E1 and Test in the context E1). 


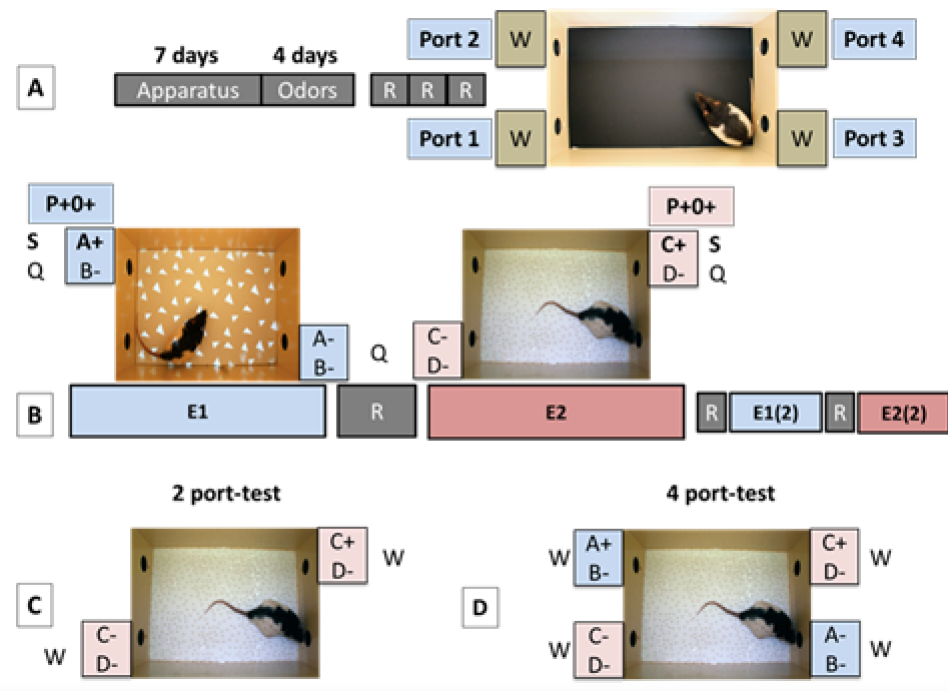

Figure 3. The different phases of the protocol. A. Shaping: Rats are submitted to 11 sessions ( 1 per day) to habituate them to the cage and the pipette operating system (a nose poke into any of the four odor ports triggers, after a delay, a pipette delivering water (Apparatus shaping, $7 \mathrm{~d}$ ). During a second phase of shaping, rats are also habituated to receive odor stimulations associated with sugar or quinine from the pipettes (Odors shaping, $4 \mathrm{~d}$ ). Once shaping is completed, rats undergo 3 routine sessions ( $R, 1$ session a day) with no odor, no enriched context, and only water available from the 4 ports. B. Episodes exposure: During each of these sessions (1 per day) the animals are exposed to 2 distinct episodes [left, Episode 1 (E1) configuration; right, Episode 2 (E2) configuration] with a $1 \mathrm{~d}$ routine $(R)$ session in between, or one day rest in home cages with maintained water deprivation. Episodes are presented once or twice in independent groups of rats. They were each characterized by a unique combination of odor-place-context associations rewarded with sugar solution (S): odor $A$ at port 2 for $E 1$; odor $C$ at port 4 for $E 2$, while the 3 other incorrect odor-place associations were associated with quinine solution (Q): odor $B$ at port 2 and odors $A$ and $B$ at port 3 for $E 1$; Odor $D$ at port 4 and odors $C$ and $D$ at port 1 for E2. During episode exposures, in a given context ("in which context"), rats encoded at which port location ("where"), one of the odors ("what") was associated with sugar solution. This configuration is referred to as $\mathrm{P}+\mathrm{O}+\mathrm{C}$ and D. Episodic memory tests. During the retrieval test $24 \mathrm{~h}$ or 24 days after the last episode session, rats are placed again in one of the context (E2, in this case) to evaluate what type of information they are able to recollect. The 2-port test (C) completely matches the episode in terms of context, odors and locations, except that only water (W) is delivered whatever place-odor configuration is experienced. The challenging 4-port test (D) is a more complex situation since it takes place in the context of E2, but with 4 accessible ports [2 previously associated with context E2 ("In context", IC, in pink) and 2 associated with context E1 ("out of context", OC, in blue)]. Each port is associated with a pair of odors that correspond to their respective episode. 
Shaping consists of two phases completed in an average of 11 daily sessions lasting 20 min at maximum. During this period, the appearance of the cage is relatively neutral (see Figure $3 \mathrm{~A}$ ): The floor is black and smooth and no odors, sounds or visual stimulation are used.

1. Phase 1 (apparatus shaping)

The objective of this phase (5-7 d) is to teach the animal how to access water in the experimental environment. It includes several steps described in details below:

Step 1: Rats housed in the same home cage are placed together into the experimental arena for 15 min with pipettes from all ports already introduced into the arena and ready to deliver water upon licking. This collective exploration period considerably reduces stress and facilitates the discovery of how to operate the drinking pipettes. This session is repeated the next day with the difference that rats are now placed into the experimental arena individually for $15 \mathrm{~min}$. Once a rat licks from the pipette, we proceed to step B2, otherwise we repeat step B1.

Step 2: At this stage, rats need to learn to trigger the introduction of the pipette into the cage by making a nose poke to the odor port. Odor ports are made as indentations into the walls of the cage, and the rats naturally come to explore them. At this stage the each pipette becomes accessible for drinking immediately after a nose-poke to the odor port and remains available for drinking for $20 \mathrm{sec}$. After this the pipette is automatically withdrawn from the cage and has to be triggered by the rat again after a refractory period (purge, duration: a few sec) which is set by the experimenter.

Note: It is better to choose a short refractory period at the beginning of shaping procedures (e.g., $5 \mathrm{sec}$ ) in order to accelerate learning of the link between the nose-poke and the pipette accessibility. For the same reason, it is important for the pipettes to be accessible for a sufficient amount of time (20 sec) to be discovered by the rats. Indeed during this period, rats usually do not wait at a particular port, but are moving around from port to port. Once they have understood how to trigger the pipettes, the amount of time during which the pipettes are accessible is reduced to 10 sec for the following session. If at this stage a rat still does not know how to trigger the pipette, this step can be repeated. This learning unit is acquired once rats perform 10-20 trials (pipette triggering) in $20 \mathrm{~min}$.

Step 3: A 3 sec offset between nose-poke and pipette entry into the cage is introduced at this stage to habituate the rats to wait for the pipette in front of the port. 10-20 trials should be initiated by the rats in a 20 min session.

Any of the 3 steps can be repeated for individual rats depending on their performance.

2. Phase 2 (odor shaping)

The objective of this phase is to habituate the animals to receive odor stimulations after the nose poke and use it as an indication for the nature of the drinking solution that will be delivered a few seconds later. During this phase, two different odors are used: Eugenol and Geraniol. Hereby, geraniol is always associated with sugar solution, 
while eugenol is always announcing the distribution of a bitter quinine solution. For a given trial, the same odor-solution combination is delivered in the four ports present in the experimental arena. Each daily session consisted of 15 to a maximum of 24 trials initiated by the rats in $20 \mathrm{~min}$. The session consists of a sequence of pseudo-randomly distributed geraniol and eugenol trials and is repeated on four consecutive days. The 3 sec delay between a nose-poke coupled with odor release and delivery of the pipette gives the animal some time to process the odor without being distracted by the pipette moving into the cage. Geraniol and eugenol are used uniquely in this shaping procedure.

During this phase, the rats progressively learn that the odor coming from the odor port can be predictive of a positive or negative reward. As a consequence, their behavioral response is modified and they begin to avoid licking the quinine solution on the basis of eugenol cue. Depending on the rat, the odor shaping phase will take from 3 to a maximum of $4 \mathrm{~d}$ before such behavioral change is observed.

Note: The first three trials of every shaping session are always positively reinforced in order to ensure that the animal receives sugar before quinine drinking solution and remains motivated throughout the session.

\section{Episodic task}

1. Routine (Figure 3A)

Prior to exposure to the episodes, rats undergo routine sessions for 3 consecutive days, with one session per day (Figure 3A). Each session consists of 12-24 trials performed in the same condition as step 3 of phase 1 of shaping. Routine sessions are thus characterized by a contextual environment with which rats are already familiar from shaping procedures (smooth black floor, no specific acoustic and visual ambiance), without any odors being released from the odor ports and with water as the only drinking solution. The objectives of the routine sessions are: First, to verify that there is an even licking distribution among the ports without any preference for a particular place; second, to further monitor and adjust water deprivation (especially when there is a delay between the shaping and the experimental phase due to e.g., some surgical procedures); and finally, to increase the memorability of the following episodes, by contrasting them to accustomed and highly familiar routine sessions.

2. Episodes (Figure 3B)

Three essential characteristics distinguish episodes from routine sessions:

a. The context: The simple black and smooth floor of the cage used during shaping and routine sessions is replaced by various materials specific to each episode. Distinct visual patterns are displayed on the floor of the arena by a projector to increase discrepancy between different episodes. Acoustic ambiance is enriched through the playing of natural sounds or music through the loudspeakers. For example, Episode 1 is characterized by a smooth white floor, projection of white 
triangles on a dark background and sounds of crickets. Episode 2 is characterized by a granular white floor, projection of white background with black circles and sounds of birds singing in the rain.

b. Only two of the four ports of the cage can be activated by the rat in each episode, ports 2 and 3 for E1, and ports 4 and 1 for E2 (Figure 3). When a rat makes a nose poke to any of the two remaining non-active ports, nothing happens.

c. On the active ports of a given episode two novel odors are delivered. For simplicity, odor A and B in Episode 1 and odors C and D in Episode 2. As a consequence, in each episode, four different combinations of odor-place can be encountered. Among these possibilities, only one odor-place combination is rewarded with sugar solution whereas the three others are associated with quinine. For example, in E1, sugar will be delivered when odor $A$ is presented in port 2 (referred to as $\mathrm{P}+\mathrm{O}+$, positively reinforced place and odor). When odor $\mathrm{B}$ is presented on the same port, the animal will receive quinine (combination referred to as $\mathrm{P}+\mathrm{O}-)$. The two other combinations involving port 3 will also lead to quinine: Odor $\mathrm{A}$ on port 3 is referred as $\mathrm{P}-\mathrm{O}+$ and odor $\mathrm{B}$ on the same port as $\mathrm{P}-\mathrm{O}-$.

Each episode session consists of 12-24 trials and lasts 20-40 min. As during the routine session, the animal is free to explore the environment. Consequently, the number of trials can vary depending on the exploratory motivation of the rat. The odors are presented at the assigned ports in a pseudorandom sequence with three repetitions of the same odor-port configurations every 12 trials. Episode sessions are separated by either one routine session (R) or one day rest in home cages with maintained water deprivation. Depending on the experiments, each episode can be repeated once before the retention test, when more homogenous performance of the group is needed (e.g., when the effect of a drug is tested on episodic memory performance).

To summarize: 


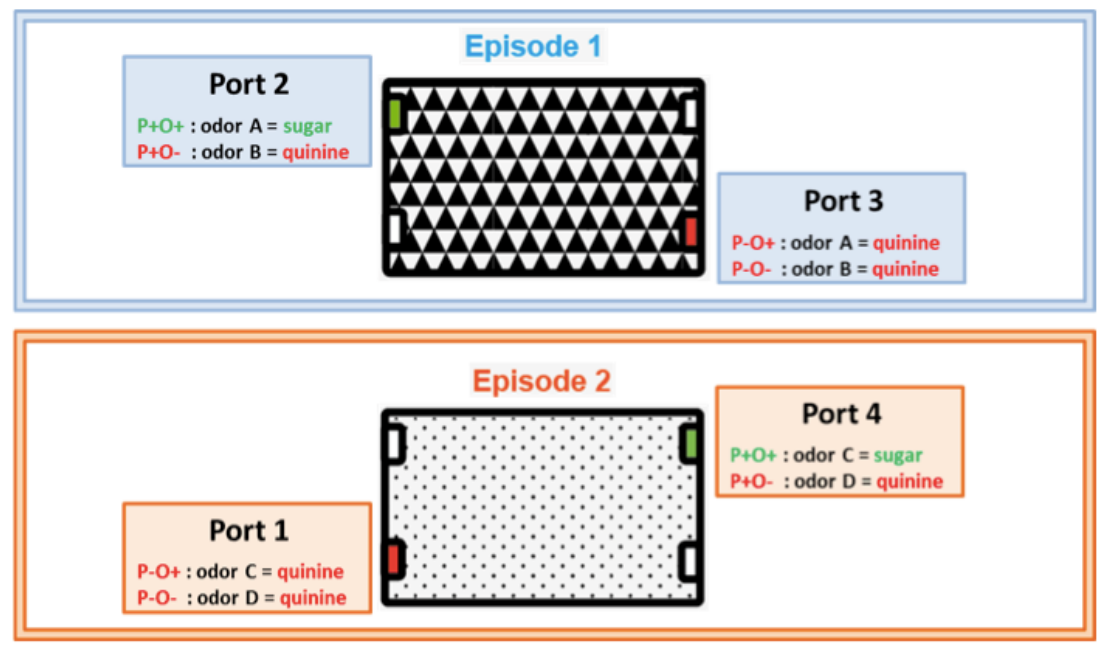

Episode 1. Odor $A\left(\mathrm{O}^{+}\right)$is associated with a sugar drinking solution at port 2 $(\mathrm{P}+\mathrm{O}+)$ and with quinine solution at port $3(\mathrm{P}-\mathrm{O}+)$. Odor $\mathrm{B}(\mathrm{O}-)$ is associated with quinine solution at port 2 ( $\mathrm{P}+\mathrm{O}-)$ and port 3 ( $\mathrm{P}-\mathrm{O}-)$.

Episode 2. Odor $\mathrm{C}\left(\mathrm{O}^{+}\right)$is associated with sugar drinking solution at port 4 $(\mathrm{P}+\mathrm{O}+)$ and with quinine solution at port $1(\mathrm{P}-\mathrm{O}+)$. Odor $\mathrm{D}(\mathrm{O}-)$ is associated with quinine solution at port 4 ( $\mathrm{P}+\mathrm{O}-)$ and port 1 (P-O-).

3. Memory recall test (Figure $3 \mathrm{C}$ or $2 \mathrm{D}$ )

The test can be performed $24 \mathrm{~h}$ or 24 days after the last exposure to Episode 2 to probe recent or remote long-term memory, respectively. In both tests, quinine and sugar are replaced by water and performance of the rats is estimated by taking into account only the 12 first trials of the test. Two versions of the test that differ in their levels of difficulty can be carried out.

2-Port Test: The test situation matches completely Episode 2 (Figure 3C). The same contextual environment as during the episode exposure is present. The same two ports are accessible and deliver the same two odors as during the corresponding episode (odors $C$ and $D$, at ports 1 and 4).

4-Port Test: The test takes place in the context corresponding to Episode 2 (same visual, auditory and tactile information), but this time all 4 ports are accessible for drinking, each delivering the same odors as during the corresponding episodes (Figure 3D). As during exposure to episode 2, ports 1 and 4 are releasing odors $C$ and $\mathrm{D}$ and are therefore referred to as "In context" (IC) combinations, additionally ports 2 and 3 are releasing odors $A$ and $B$, as during exposure to episode 1 and are referred to as "out of context" (OC) combinations.

Note: One modification introduced to the original protocol is the removal of the routine sessions between the episodes. Instead of routine sessions the rats are kept in their home cages on the same water deprivation schedule as used before the shaping 


\section{bĭ̈-protocol}

http://www.bio-protocol.org/e1740 Vol 6, Iss 5, Mar 5, 2016

procedures ( $\sim 30$ min access to water in the morning and evening). We didn't notice a difference in performance between the two protocols (with either a routine session between the episodes, or one day rest in the home cage with maintained water deprivation) and recommend therefore the simpler version of the protocol which doesn't include routine sessions between the episodes.

Note: The level of difficulty of both tests can be additionally adjusted by using more overlapping or distinct contextual features, such as e.g. the flooring material of the experimental cage, or the chemical proximity of the odors.

\section{Analysis}

The output files of the experiments contain the data about the total amount of licks done for each odor-port configuration on every single trial.

1. To normalize the measures of licking behavior across rats, the licks of all the trials that correspond to a specific odor-port configuration (e.g. $\mathrm{P}+\mathrm{O}+$ ) are summed up for a given rat and divided by the total amount of licks made by this rat in the respective session (lick ratio).

2. The group performance is determined by calculating the group mean of the ratios (calculated for each individual rat in step B1) by odor-port configuration, which is referred to as the licks index.

3. The same calculations can be performed by analyzing the number of times rats encountered each of the different odor-port configurations (referred to as visits). For this, trials performed on a given odor-port configuration are summed up and divided by all the trials made in a session for each individual rat, from which the group mean performance is calculated.

\section{$\underline{\text { Representative data }}$}

Figure 4 depicts a group performance of 6 rats that experienced two exposures to episodes E1 and E2 according to the experimental protocol described above. Recollection memory of Episode 2 was tested after a retention interval of $24 \mathrm{~h}$ in a 2-port test. As a group, rats remembered the correct, combined odor-place information linked to the context of the previously experienced E2 episode, since they were making significantly more licks at $\mathrm{P}+\mathrm{O}+$ configuration of the $\mathrm{E} 2$ context compared to all other odor-port configurations. Nonparametric Friedman test, followed by Wilcoxon test was used for statistical analysis $\left({ }^{*} \mathrm{p}<0.05\right)$. 


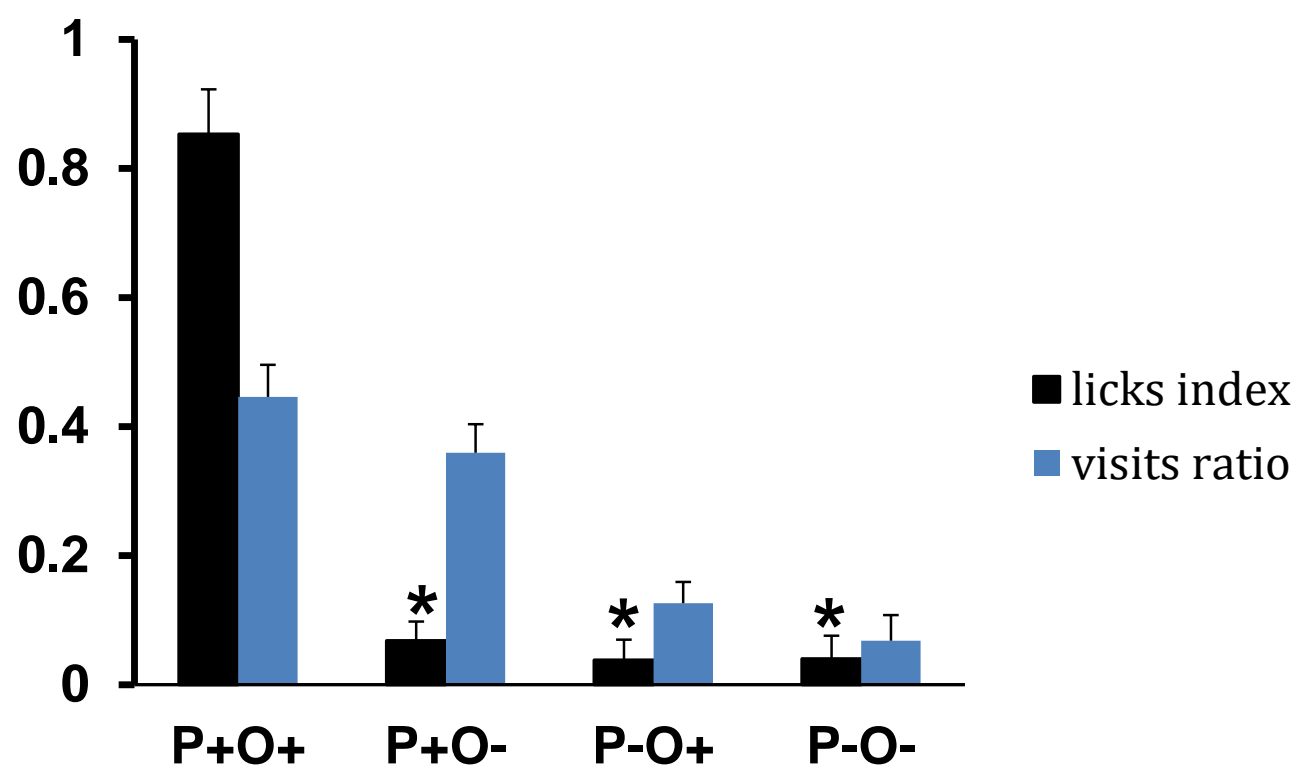

Figure 4. Group memory performance in a 2-port test. $24 \mathrm{~h}$ after two exposures to the episodes. In the group as a whole there are more visits to positively reinforced port $\mathrm{P}+$, which delivered sugar during the initial exposure to the episode, compared to $\mathrm{P}$ - where no sugar is delivered irrespective of the odor (visits ratio: blue bars). Despite the fact that during the test session water is delivered in every encountered configuration, the group shows a significantly higher lick ratio for the previously positively reinforced odor-port configuration $(\mathrm{P}+\mathrm{O}+)$ compared to the three other odor-port configurations in the given context (lick ratio: black bars) ( $\left.{ }^{\star} \mathrm{p}<0.05\right)$. Figure adapted from Veyrac et al. (2015). As an illustration, Videos 1 and 2 are provided below.

Video 1. Example of a rat exhibiting a drinking behavior. The animal puts his nose into the odor port, waiting the release of the pipette and drinks until the end of the trial.

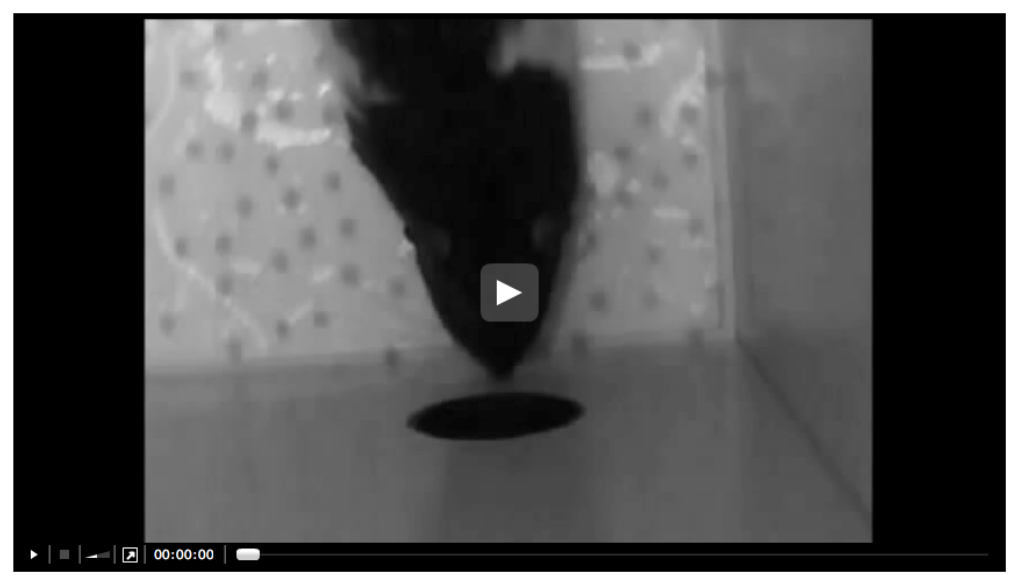


Video 2. Example of a rat with an avoidance behavior. The animal puts his nose into the odor port, samples the odor associated with quinine and avoids drinking.

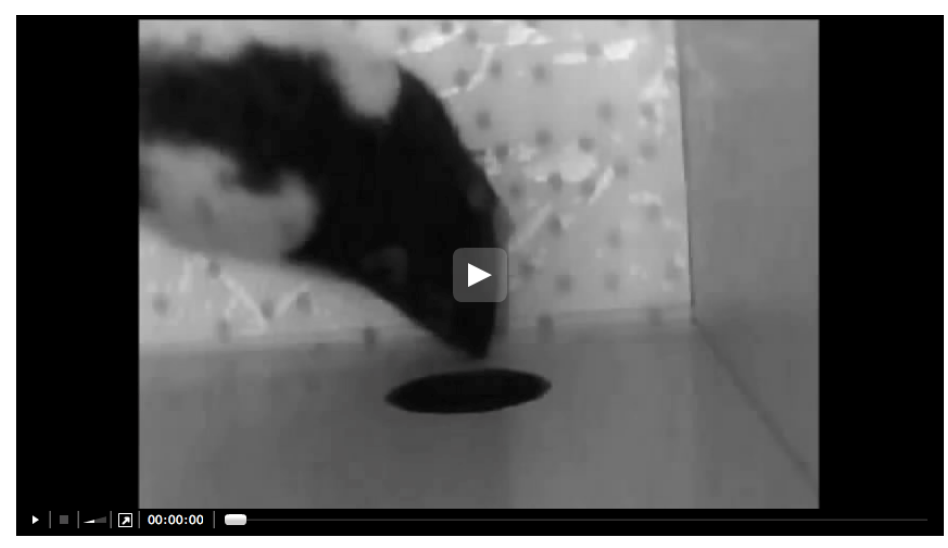

Beside the group analysis, individual results are also considered to determine the memory recall profile of each animal according to respective test situation. Two examples are presented in Figure 5. These individual profiles allow classification of each animal according to the type of information it is able to retrieve in the different test situations.

A. "what-where" rat

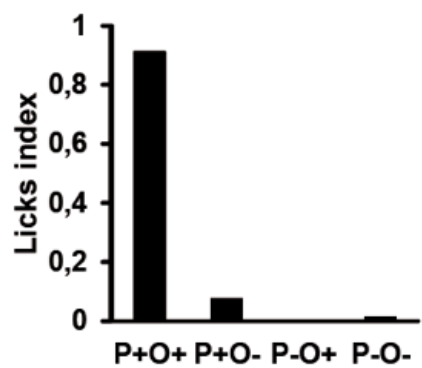

B. "where" rat

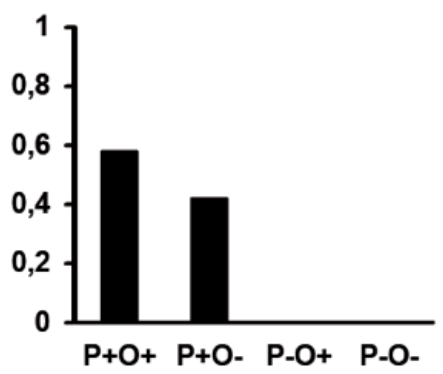

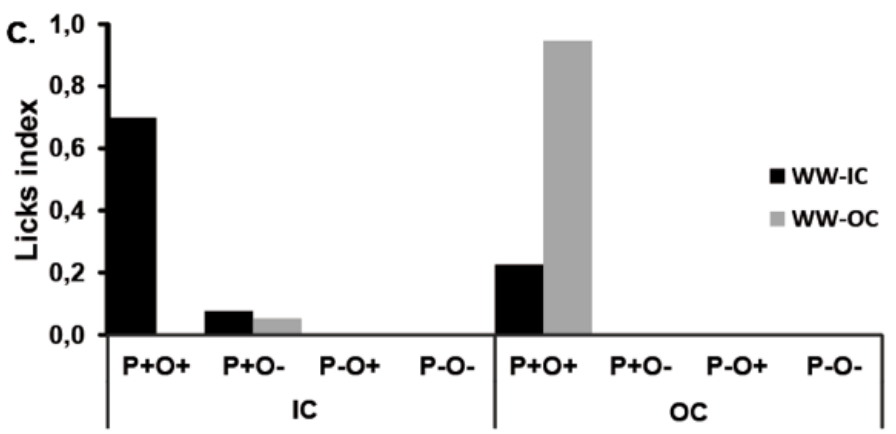

Figure 5. Examples of licking profiles for 4 different rats during the recall test of episode 2, in a 2-port (A and B) and a 4-port test condition (C). A. Performance of a rat presenting a typical "What-Where" profile, This rat drinks more on the good port associated with the tested context and with the correct odor $(\mathrm{P}+\mathrm{O}+)$, thus showing a good episodic recollection. B. Performance of a rat presenting a typical "Where" profile. This rat drinks on the good port associated with the tested context $(\mathrm{P}+)$ but does not exhibit any discrimination between the two odors, thus showing a good memory only for the place information associated with this episode. C. The 4-port test situation allows it to test 
explicitly if the animal is able to retrieve the whole episode. Examples of two different memory profiles observed in the 4-port test performed in the context of episode 2. WW-IC: this rat is categorized as "What-Where-in context", the rat is licking mainly at the $\mathrm{P}+\mathrm{O}+$ configuration of the port which delivered sugar in the tested context of episode 2. WW-OC: This rat is categorized as "What-Where-out of context", the rat is licking at the $\mathrm{P}+\mathrm{O}+$ configuration corresponding to episode 1 that was not currently tested.

\section{Acknowledgments}

The protocol presented here is adapted from Veyrac et al. (2015). This work was supported by CNRS, University Lyon 1, University Paris-Sud 11, and by grants from the "Agence Nationale de la Recherche" (ANR-2010-BLAN-1413-01) to SL and NR, from the "Fondation pour la recherche médicale" (FDT20140930863) to AG and from LABEX CORTEX (ANR-11-LABX-0042) to NR.

\section{$\underline{\text { References }}$}

1. Courtiol, E., Lefevre, L., Garcia, S., Thevenet, M., Messaoudi, B. and Buonviso, N. (2014). Sniff adjustment in an odor discrimination task in the rat: analytical or synthetic strategy? Front Behav Neurosci 8: 145.

2. Herlitz, A. and Viitanen, M. (1991). Semantic organization and verbal episodic memory in patients with mild and moderate Alzheimer's disease. J Clin Exp Neuropsychol 13(4): 559-574.

3. Martin, C., Gervais, R., Hugues, E., Messaoudi, B. and Ravel, N. (2004). Learning modulation of odor-induced oscillatory responses in the rat olfactory bulb: a correlate of odor recognition? J Neurosci 24(2): 389-397.

4. Sezille, C., Messaoudi, B., Bertrand, A., Joussain, P., Thevenet, M. and Bensafi, M. (2013). A portable experimental apparatus for human olfactory fMRI experiments. $J$ Neurosci Methods 218(1): 29-38.

5. Torquet, N., Aime, P., Messaoudi, B., Garcia, S., Ey, E., Gervais, R., Julliard, A. K. and Ravel, N. (2014). Olfactory preference conditioning changes the reward value of reinforced and non-reinforced odors. Front Behav Neurosci 8: 229.

6. Veyrac, A., Allerborn, M., Gros, A., Michon, F., Raguet, L., Kenney, J., Godinot, F., Thevenet, M., Garcia, S., Messaoudi, B., Laroche, S. and Ravel, N. (2015). Memory of occasional events in rats: individual episodic memory profiles, flexibility, and neural substrate. J Neurosci 35(19): 7575-7586. 